\title{
Pneumonia in young adults with asthma: impact on subsequent asthma exacerbations
}

This article was published in the following Dove Press journal:

Journal of Asthma and Allergy

\author{
Stephen I Pelton ${ }^{1,2}$ \\ Kimberly M Shea' \\ Rebecca Bornheimer ${ }^{3}$ \\ Reiko Sato ${ }^{4}$ \\ Derek Weycker ${ }^{3}$ \\ 'Department of Epidemiology, Boston \\ University Schools of Medicine and Public \\ Health, Boston, MA, USA; ${ }^{2}$ Maxwell \\ Finland Laboratories, Boston Medical \\ Center, Boston, MA, USA; ${ }^{3}$ Policy \\ Analysis Inc. (PAI), Brookline, MA, USA; \\ ${ }^{4}$ Health Economic \& Outcomes \\ Research, Pfizer Inc., Collegeville, \\ PA, USA
}

Background: Recent studies of community-acquired pneumonia (CAP) have recognized acute cardiac complications - such as myocardial infarction, arrhythmia, or congestive heart failure ( $\mathrm{CHF}$ ) — as frequent complications during the acute process. As well, a prolonged vulnerability to exacerbations of underlying comorbidities - such as CHF and COPD - has been observed following CAP. We hypothesized that young adults with underlying asthma could also be adversely impacted over a prolonged time period following CAP.

Methods: Using a retrospective matched-cohort design and data from a US private healthcare claims repository ( $>15 \mathrm{M}$ persons annually), we selected all adults 18-49 years of age with evidence of asthma as their only comorbidity for inclusion in the source population. Then, from the source population, we matched one comparison patient to each CAP patient based on index date, age, sex, and selected markers for health status (eg, history of asthmarelated healthcare encounters), and evaluated subsequent outpatient and inpatient encounters for asthma exacerbations.

Results: Asthma exacerbations were identified twice as often in the 12 months subsequent to acute CAP. Cumulative incidence proportions for asthma exacerbations requiring hospitalization or emergency department care after 12 months of follow-up were $19.9 \%$ for those previously hospitalized with CAP versus $9.0 \%$ for matched comparison patients (difference, $10.9 \%$; $p<0.001$ ), and were $12.4 \%$ for non-hospitalized CAP patients versus $7.7 \%$ for matched counterparts (difference, $4.7 \% ; p<0.001$ ).

Conclusion: Our analysis provides further evidence that acute CAP has a prolonged impact on respiratory health.

Keywords: pneumonia, asthma, disease exacerbation, disease progression

\section{Introduction}

Many investigators, including ourselves, have observed a link between recurrent wheezing or asthma and increased incidence of invasive pneumococcal disease (IPD) in both children and adults, although the mechanisms that increase risk are uncertain. ${ }^{1}$ In 18-49 year-old adults with asthma, an age group more likely to have extrinsic disease, we reported a nearly three-fold increase in IPD incidence compared with age-matched, healthy counterparts. ${ }^{2}$ The increased risk of IPD has also been associated with age and asthma severity, with the relative risk as large as 6.6 for children less than 5 years of age with severe asthma compared to age-matched, healthy children. ${ }^{3} \mathrm{We}$ also observed that children and adults with asthma had more than a three-fold increased incidence of all-cause pneumonia, and a comparable increased risk for pneumococcal pneumonia. ${ }^{2}$ Although some argue that the increase in
Correspondence: Derek Weycker Policy Analysis Inc. (PAI), Four Davis Court, Brookline, MA 02445, USA

$\mathrm{Tel}+\mathrm{I} 6172324400$

Email dweycker@pai2.com 
"pneumonia" is confounded by atelectasis, we think the observed increase in IPD supports the likelihood that persons with asthma are at increased risk for pneumococcal pneumonia and potentially other pneumonia pathogens.

Peyrani and Ramirez recently reported that adults surviving acute episodes of community-acquired pneumonia (CAP) were at greater risk of death during the subsequent year, long after the acute event. ${ }^{4}$ We previously reported that adults with congestive heart failure (CHF) or COPD had more frequent exacerbations of their underlying disease in the 12 months following acute CAP episodes relative to matched comparison patients without CAP. ${ }^{5}$ Accordingly, we hypothesized that, in addition to an increased incidence of all-cause pneumonia as demonstrated in previous studies, ${ }^{2,3}$ adults with asthma would also have increased rates of exacerbations of their disease following recovery from an acute CAP episode.

\section{Methods}

\section{Study design and data source}

A retrospective matched-cohort design and data from a US private healthcare claims repository $(>15 \mathrm{M}$ persons annually) were employed. A detailed description of our methodological approach and data source has been previously reported. ${ }^{5}$

\section{Ethics approval and consent to participate}

The data extract was de-identified prior to its release to study investigators, and thus its use for health services research is compliant with the HIPAA Privacy Rule and federal guidance on Public Welfare and the Protection of Human Subjects, and IRB status is exempt.

\section{Source and study populations}

The source population comprised patients aged $\geq 18$ years who, between January 2009 and June 2013, had evidence of asthma based on $\geq 1$ acute-care inpatient diagnosis (ICD-9 493.XX), or $\geq 2$ ambulatory diagnoses (separated by $\geq 30$ days) plus $\geq 1$ filled prescription for inhaled albuterol. From the source population, beginning in January 2010 and on a month-bymonth basis thereafter, patients who had continuous health benefits, evidence of asthma (as defined above), and no evidence of pneumonia during the prior year, and who had an incident CAP episode in that month, were identified. CAP episodes were ascertained based on diagnosis codes, procedure codes, and drug codes, and were stratified by care setting (hospital vs ambulatory); patients requiring inpatient care who died or spent $>30$ days in hospital were excluded. The date of the initial CAP encounter plus 30 days was defined as the index date.

One comparison patient was matched to each CAP patient based on index date, age $( \pm 1$ year $)$, sex, and selected markers for health status (eg, history of asthmarelated healthcare encounters, comorbidity profile). Like CAP patients, comparison patients were required to have continuous health benefits, evidence of asthma, and no evidence of pneumonia during the year prior to the index date. Once matched, both the CAP patient and comparison patient were included in the study population and removed from the source population. The same process was repeated for each subsequent calendar month ending in May 2013.

\section{Measures and analyses}

Asthma exacerbations were ascertained over the 1-year period following the index date, and were defined as a hospitalization with a principal diagnosis of asthma or an emergency-department (ED) visit with an asthma diagnosis in any position. Cumulative incidence proportions for asthma exacerbations were compared between CAP patients and matched comparison patients using the McNemar test. Base-case analyses were restricted to asthma patients aged 18-49 years without other chronic conditions because chronic obstructive pulmonary disease (COPD), which has a different pathogenesis, is often misclassified as asthma in older adults. For purposes of comparison, however, sensitivity analyses were conducted for adults aged 18-49 years with asthma and $\geq 1$ comorbidity.

\section{Results}

\section{Patient characteristics}

Among the $1.2 \mathrm{M}$ asthma patients aged $\geq 18$ years in the source population, 28,957 (2.4\%) developed CAP during the 3.5 year observation period and were matched to comparison patients. Among the 28,957 matched pairs, 4,048 were aged 18-49 years and had no other chronic conditions, and thus were included in base-case analyses. One-third of CAP episodes required hospitalization. Among hospitalized CAP patients and their 
matched counterparts, 62\% were aged 35-49 years and $11 \%$ had an asthma-related hospitalization in the prior year; other characteristics and duration of followup were largely comparable. Characteristics of ambulatory CAP patients and their matched counterparts were similar.

Asthma Exacerbations Requiring Hospitalization among Hospitalized CAP Patients and Matched Comparison Patients

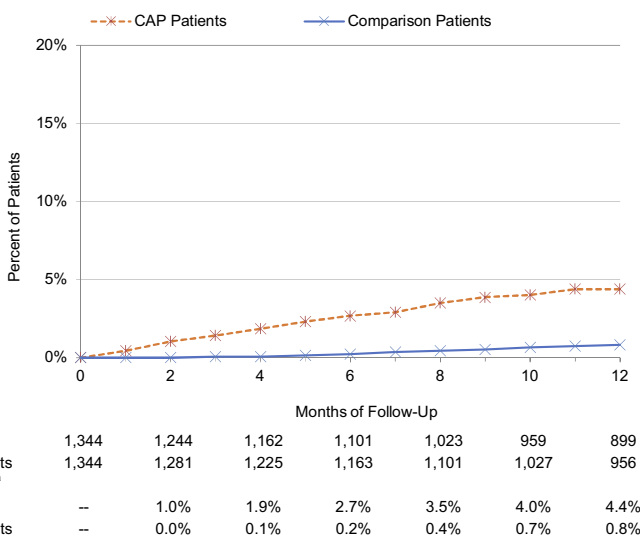

Asthma
Patients

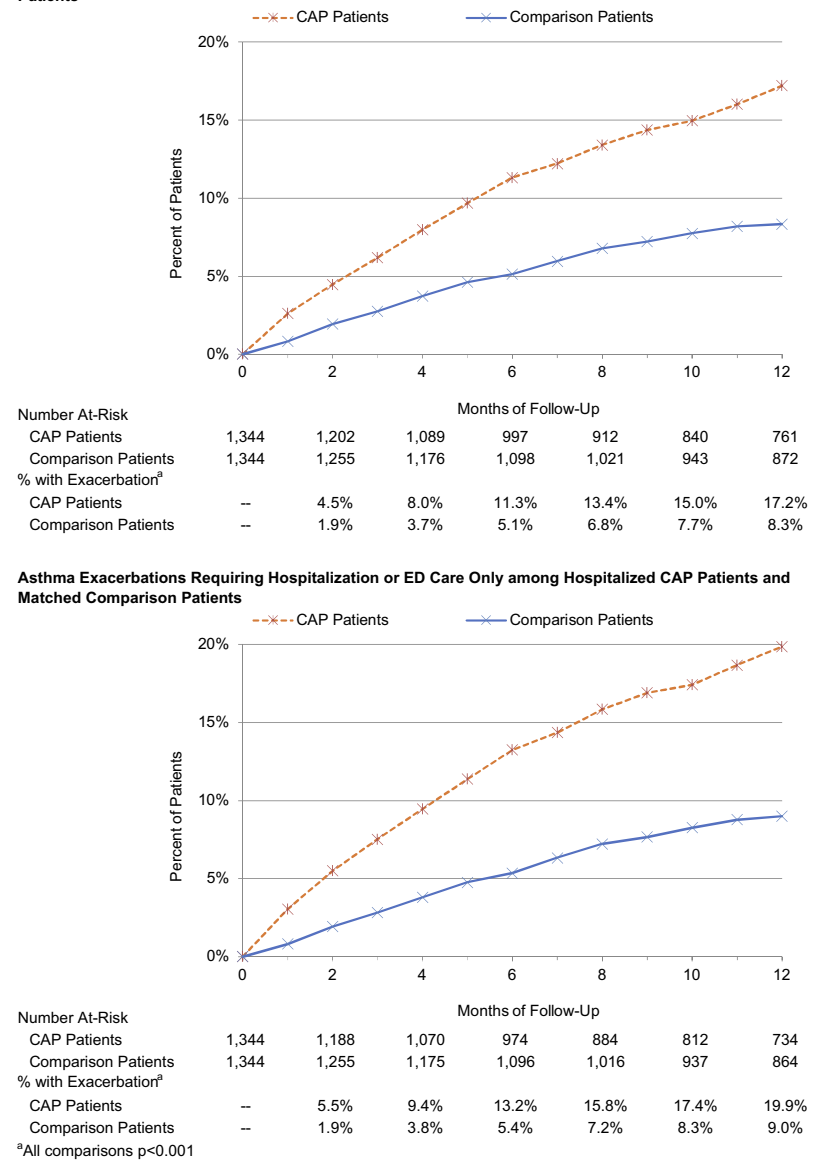

\section{Asthma exacerbations}

Cumulative incidence proportions for asthma exacerbations-including those requiring hospitalization or ED care only - at month 12 of follow-up were $19.9 \%$ for hospitalized CAP patients versus $9.0 \%$ for matched comparison patients (difference, $10.9 \% ; p<0.001$ ), and $12.4 \%$

Asthma Exacerbations Requiring Hospitalization among Ambulatory CAP Patients and Matched Comparison Patients

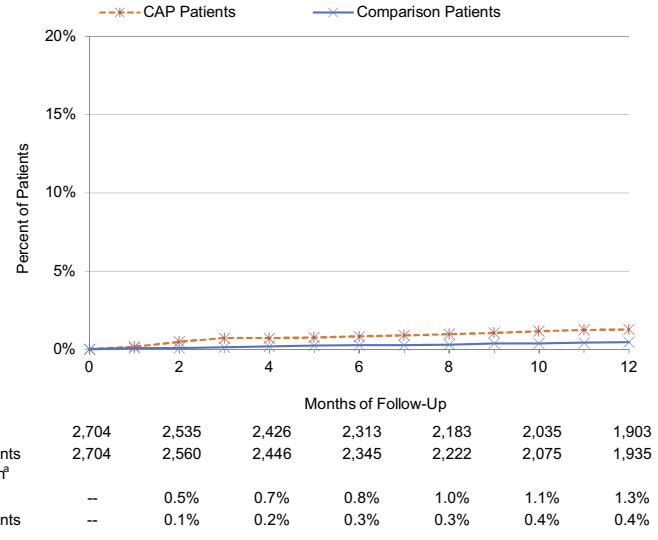

Patients

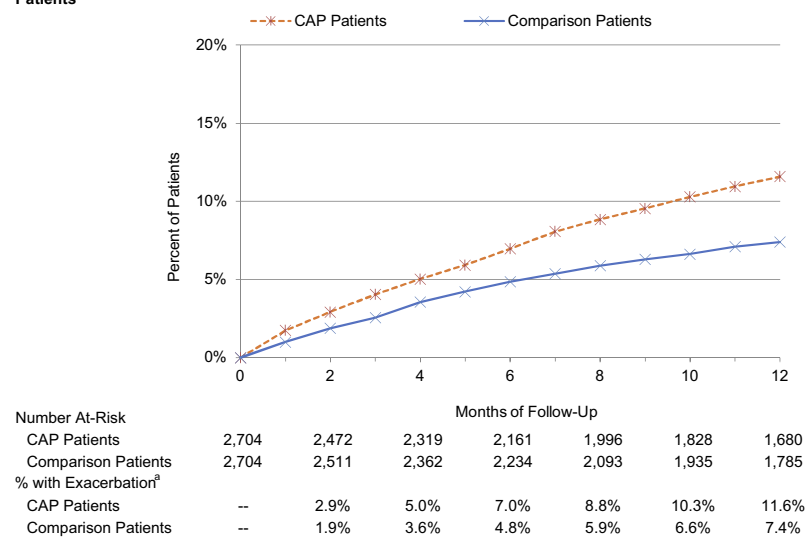

Asthma Exacerbations Requiring Hospitalization or ED Care Only among Ambulatory CAP Patients and Matched Comparison Patients

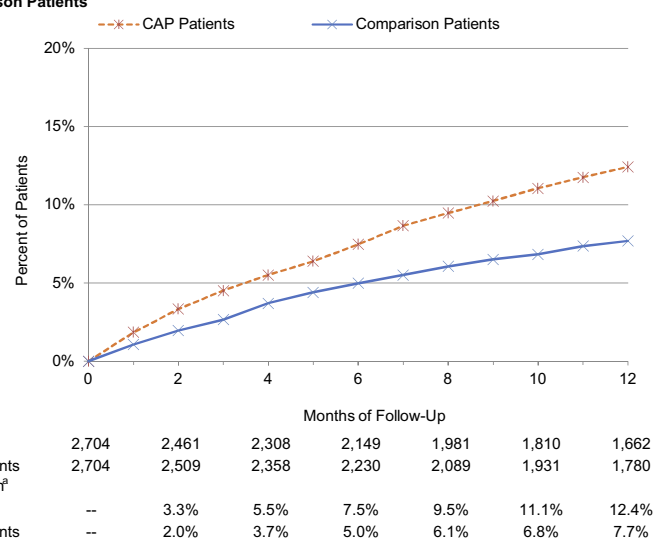

Figure I Cumulative incidence proportions for asthma exacerbations among CAP patients and matched comparison patients aged I8-49 years without other chronic conditions (ie, other than asthma), by care setting of CAP episode (hospital vs ambulatory) and care setting of asthma exacerbation (hospital vs ED).

Abbreviation: CAP, Community-acquired pneumonia. 
for ambulatory CAP patients vs $7.7 \%$ for matched counterparts (difference, $4.7 \%$; $p<0.001$ ) (Figure 1). For both the hospitalized and ambulatory subgroups, differences in asthma exacerbations between CAP patients and comparison patients persisted from month 2 through the end of the 12-month observation period, and interval differences between each 2-month period demonstrated that the increased risk of asthma exacerbations persisted for at least the subsequent 12 months following the CAP episode. Results among adults aged 18-49 years with asthma and $\geq 1$ comorbidity were comparable.

\section{Discussion}

Our analysis suggests that acute CAP increases the one year incidence of asthma exacerbations among adults 18-49 years of age. Asthma exacerbations were identified twice as often in the 12 months subsequent to acute CAP, irrespective of care setting (ie, following outpatient or hospitalized CAP), relative to matched comparison patients. The need for hospitalization, perhaps a marker of severity, at the initial respiratory event was linked to the risk of further exacerbations; those hospitalized had a greater risk $(19.9 \%$ vs $12.4 \%)$ of subsequent exacerbations requiring ED visits or hospital admissions compared to those initially managed in the ambulatory setting. The difference in exacerbations between CAP and comparison groups was evident by month 2 after the acute CAP episode, and persisted for the entire follow-up period. The excess in ED visits or hospitalizations was present for both the group in which asthma was the single comorbidity as well as those who had additional comorbidities (data not reported).

CAP is associated with increased local and systemic cytokine responses, specifically pro inflammatory cytokines that attract and activate neutrophils as part of the host defense mechanisms. ${ }^{6}$ Pro-inflammatory cytokines (IL-6, IL-12, IL-17, and IL-18) are important mediators in the innate response to pneumococci and elevated concentrations often persist beyond the acute phase of pneumonia, especially IL-6. Pro-inflammatory cytokines, such as TNF- $\alpha$, IL-1 $\beta$, and IL- 6 , are found in increased amounts in the sputum and BAL fluid in individuals with asthma, and are credited with enhancing inflammation and airway reactivity supporting the potential interaction between CAP and subsequent asthma exacerbations. ${ }^{7}$

Other pulmonary conditions, such as bronchiectasis, also have been reported to be associated with asthma exacerbations. For example, Kang and colleagues reported a threefold increase in exacerbations in patients with asthma and bronchiectasis compared to asthma only. ${ }^{8}$ He hypothesized that chronic airway inflammation or possible persistent airway colonization could be triggers for the exacerbations. Peyrani and Ramirez suggest the long-term mortality following CAP may be linked to a persisting inflammatory response, resulting in "inflammaging.", They cite persistent elevated levels of cytokines at the time of hospital discharge as evidence of the potential for prolonged local inflammation. Support for a role for such chronic inflammation is suggested by the success of long term azithromycin in reducing asthma exacerbations in adults, albeit there may be other mechanisms by which azithromycin is effective. ${ }^{9}$

The limitations of our analysis are related to use of a healthcare claims database to identify and match patients with asthma and CAP to individuals with asthma but without CAP. Although subjects were matched on demographic features and asthma-related encounters in the prior year, it would have been preferable (but not possible) to match on physiologic measures of pulmonary function. As well, the use of healthcare claims data does not allow us to match on smoking status.

Our findings support the increasing evidence that CAP is not simply an acute event with complete resolution but leaves a residual impact on the risk of disease exacerbations over a prolonged period of time. As with patients with comorbidities such as CHF and COPD, our findings also support the prioritization of pneumonia prevention strategies among adults with asthma, including use of the 23 -valent pneumococcal polysaccharide vaccine and annual influenza vaccine, both of which are currently recommended for all individuals aged 18-64 years with asthma. ${ }^{10}$

\section{Data sharing statement}

There are restrictions on data sharing. The study used healthcare claims data to evaluate patients with asthma, and to determine the impact of CAP on their underlying comorbidity. The healthcare claims database (Truven Health Analytics MarketScan ${ }^{\circledR}$ Databases) is proprietary, provided by a thirdparty vendor, and the authors do not have permission to disseminate this data without vendor approval. The study sponsor has purchased access to the Truven MarketScan Database (on a contract per-project use). Access to this data set is available to any other interested parties for a fee set by Truven Health Analytics (http://truvenhealth.com/your-healthcare-focus/ana lyticresearch/marketscan-research-databases).

\section{Acknowledgments}

Funding for this research was provided by Pfizer Inc. to Policy Analysis Inc. (PAI). The study sponsor, Pfizer Inc., 
reviewed the study research plan and study manuscript; data management, processing, and analyses were conducted by PAI. All final analytic decisions and the decision to submit for publication were made exclusively by study investigators. There are no patents, products in development, or marketed products to declare.

\section{Author contributions}

Authorship was designated based on guidelines promulgated by the International Committee of Medical Journal Editors (2004). All persons who met criteria for authorship were listed as authors on the title page. The contribution of each of these persons to this study is as follows: (1) conception and design (all authors), acquisition of data (Sato, Weycker), analysis or interpretation of data (all authors); and (2) preparation of manuscript (all authors), critical review of manuscript (all authors). All authors have read and approved the final version of the manuscript, and agreed to be accountable for all aspects of the work.

\section{Disclosure}

Kimberly M Shea was employed by Boston University School of Public Health during the conduct of this research and Stephen I Pelton was employed by Boston University Schools of Medicine and Public Health, both received financial support from Pfizer Inc. for their participation in study design, data analysis, and data interpretation. Kimberly $\mathrm{M}$ Shea has received a previous investigator-initiated research grant from Pfizer Inc. Stephen I Pelton has served as an advisory board member for, and received investigator-initiated research grants from Pfizer Inc. and other vaccine manufacturers. Rebecca Bornheimer and Derek Weycker are employ- ees of Policy Analysis Inc. (PAI), which received financial support from Pfizer Inc. for this study (including manuscript preparation). Reiko Sato is employed by and owns stock in Pfizer Inc. The authors report no other conflicts of interest in this work.

\section{References}

1. Talbot TR, Hartert TV, Mitchel E, et al. Asthma as a risk factor for invasive pneumococcal disease. $N$ Engl J Med. 2005;352:2082-2090. doi:10.1056/NEJMoa044113

2. Shea K, Edelsberg J, Weycker D, Farkouh R, Strutton D, Pelton S. Rates of pneumococcal disease in adults with chronic medical conditions. Open Forum Infect Dis. 2014. doi:10.1093/ofid/ofu024

3. Pelton SI, Weycker D, Farkouh RA, Strutton DR, Shea KM, Edelsberg J. Risk of pneumococcal disease in children with chronic medical conditions in the era of pneumococcal conjugate vaccine. Clin Infect Dis. 2014;59(5):615-623. doi:10.1093/cid/ciu348

4. Peyrani P, Ramirez JA. One-year mortality in patients with community-acquired pneumonia. Univ Louisville J Respir Infect. 2017;1:44-49.

5. Bornheimer R, Shea KM, Sato R, Weycker D, Pelton SI. Risk of exacerbation following pneumonia in adults with heart failure or chronic obstructive pulmonary disease. PLoS One. 2017;12(10): e0184877. doi:10.1371/journal.pone. 0184877

6. Bordon J, Aliberti S, Fernandez-Botran R, et al. Understanding the roles of cytokines and neutrophil activity and neutrophil apoptosis in the protective versus deleterious inflammatory response in pneumonia. J Infect Dis. 2013;17(2):e76-e83.

7. Barnes PJ. The cytokine network in asthma and chronic obstructive pulmonary disease. J Clin Invest. 2008;118(11):3546-3556. doi:10.1172/JCI36130

8. Kang HR, Choi GS, Park SJ, et al. The effects of bronchiectasis on asthma exacerbation. Tuberc Respir Dis. 2014;77:209-214. doi:10.4046/trd.2014.77.5.209

9. Gibson PG, Yan IA, Upham JW, Effect of azithromycin on asthma exacerbations and quality of life in adults with persistent uncontrolled asthma (AMAZES): a randomised, double-blind, placebo-controlled trial. Lancet. 2017;390:659-668. doi:10.1016/S0140-6736(17)31281-3

10. Centers for Disease Control and Prevention. Immunization Schedules. Available from: https://www.cdc.gov/vaccines/schedules/easy-to-read /adult-conditions-easyread.html. Accessed January 30, 2019.
The Journal of Asthma and Allergy is an international, peer-reviewed open-access journal publishing original research, reports, editorials and commentaries on the following topics: Asthma; Pulmonary physiology; Asthma related clinical health; Clinical immunology and the immunological basis of disease; Pharmacological interventions and new therapies. The manuscript management system is completely online and includes a very quick and fair peer-review system, which is all easy to use. Visit http://www.dovepress.com/testimonials.php to read real quotes from published authors. 